\title{
A NOTE ON THE ALGEBRA OF BOUNDED FUNCTIONS. II
}

\author{
KENNETH G. WOLFSON
}

1. Let $K$ be a commutative $B^{*}$-algebra with identity 1 (with $\left\|k^{*} k\right\|=\|k\|^{2}$ for all $k \in K$, and $\|1\|=1$ ). Then $K$ is equivalent (isomorphic in a norm and ${ }^{*}$ preserving manner) to the algebra $C(M)$ of all continuous complex-valued functions on the compact Hausdorff space $M$ (its structure space) $[1 ; 2]$. In [5] we have given necessary and sufficient conditions that $K$ be equivalent to $B(X)$, the ring of all bounded complex-valued functions on the discrete space $X$. These conditions were ideal-theoretic, involving the annulets (annihilating ideals) of $K$, and did not depend on the representation $C(M)$. Using the representation $C(M)$ two further characterizations of $B(X)$ are given in [3], one involving the properties of the space $M$, and the other the notion of projection. The characterizations in [3] are derived independently of the one in [5], and in fact no attempt is made in [3] to relate directly the ideal-theoretic conditions with the notions used there. In this note, we show how the characterizations in [3] can be derived from the characterization in [5] by relating directly the ideal-theoretic properties of $K$ with the properties of the structure space $M$, and with the idea of projection. In particular, the lattice of annulets of $K$ is anti-isomorphic to the lattice of regular open sets in $M$ (Lemma 1). Another characterization of $B(X)$ (Theorem 4 ) is a byproduct of our procedure.

2. The notation will follow that in [5]. If $G \leqq K$ then $R(G)$ is the set of all functions $k \in K=C(M)$ such that $k g=0$ for all $g \in G$. Such ideals were called annulets. $N(G)$ is the set of $y \in M$ such that $g(y)=0$ for all $g \in G$. If $S \leqq M$, then $A(S)$ is the set of functions $f$ such that $f(x)=0$ for all $x \in S$. Since $N(G)=\bigcap_{\varrho \in G} N(g)$, it is a closed set. Now by following the arguments of Lemma 1 of [5], and using the fact that if $O$ is open in a compact space and $x \in O$, there exists a function $f \in K$ with $f(x)=1$ and $f\left(O^{\prime}\right)=0$, we have

(1) $R(G)=A\left[N(G)^{\prime}\right]$ for $G \leqq K$;

(2) $R[A(S)]=A\left(S^{\prime}\right)$ if $S$ is a closed subset of $M$;

(3) $N[A(S)]=S$ if $S$ is a closed subset of $M$.

Now (1) and (2) show that an annulet of $K$ is the set of all functions vanishing on an open set of $M$ and conversely. Since $A(S)=A(\bar{S})$

Received by the editors September 2, 1955. 
and $S \leqq$ int $\bar{S} \leqq \bar{S}$ for any open set $S$, an annulet is the set of functions vanishing on a regular open set and conversely. ( $S$ is a regular open set if $S=$ int $\bar{S}$ where int $\bar{S}$ is the largest open set in $\bar{S}$.) If $S$ and $T$ are regular open sets such that $A(S)=A(T)$, then $\bar{S}=\bar{T}$ by (3) and hence $S=$ int $(\bar{S})=\operatorname{int}(\bar{T})=T$. We have:

LEMMA 1. The lattice of annulets of $K$ is anti-isomorphic to the lattice of regular open sets in $M$.

LEMMA 2. The following are equivalent:

(1) The sum of two annulets is an annulet.

(2) Every annulet is generated by an idempotent.

(3) Every regular open set in $M$ is closed.

Proof. Assume (1) and let $A(S)$ be any annulet, where $S$ is a regular open set. Let $T=\bar{S}^{\prime}$. Then $T$ is a regular open set such that $S \cap T=0$. Now $A(S) \cup A(T)$ (the smallest annulet containing $A(S)$ and $A(T))=A(S)+A(T)$ since this is an annulet by (1). But $A(S) \cup A(T)=A(S \cap T)=A(0)=K$ by Lemma 1 . Since $A(S) \cap A(T)$ $=0$ we have $A(S) \oplus A(T)=K$, and it follows as in Theorem 3 of [5] that $A(S)=e K$ where $e$ is the characteristic function of $S^{\prime}$.

Now assume (2), let $S$ be a regular open set, and $A(S)$ the corresponding annulet. Then $A(S)=e K$, so $N(e)=N(e K)=N[A(S)]=\bar{S}$. Hence $\bar{S}$ is open and closed. Then $S=$ int $\bar{S}=\bar{S}$, and $S$ is closed.

Now assume (3) and let $S, T$ be regular open sets $(S \cap T$ is also regular). Since $S$ is open and closed, $A(S)$ is generated by the characteristic function of $S^{\prime}$. Then $A(T), A(S \cap T)$ are similarly generated by idempotents, and the proof can now be completed as in the proof of Theorem 2 of [5] to show $A(S)+A(T)$ is the annulet $A(S \cap T)$.

LEMMA 3. The following statements are equivalent:

(1) Every nonzero closed ideal of $K$ contains a minimal ideal.

(2) For each $k \neq 0$, there exists a minimal projection e such that $k e \neq 0$.

(3) The space $M$ contains a dense subset of isolated points.

Proof. Assume (1) and let $k \in K, k \neq 0$. Let $J=\overline{K k}>0$. Then $J$ contains a minimal ideal of form $K e$ for a projection $e$ [4, p. 64] since any idempotent in $K=C(M)$ is obviously self-adjoint. Now $K e$ is a simple commutative ring with identity, hence a field. By the Gelfand-Mazur theorem $\mathrm{Ke}$ is isomorphic to the complex numbers, so that $e$ is a minimal projection. If $k e=0,(K k) e=0, J e=0, e^{2}=e=0$, a contradiction, so that (2) follows.

The fact that (2) implies (3) is proven in Theorem 2 of [3].

Now assume (3), and let $J$ be a closed ideal $\neq 0$ so that $k \neq 0$, $k \in J$. If $k e \neq 0$ for some minimal projection $e$, then $k e=\lambda e$ for com- 
plex $\lambda, e=\lambda^{-1} k e \in J$ and $J$ contains the ideal $K e$ which is obviously minimal, since it is a field. Assume if possible that $k e=0$ for all minimal projections $e$. Let $X_{0}$ be the dense set of isolated points. Each minimal projection $e$ is the characteristic function of a point of $X_{0}\left[3\right.$, Theorem 2]. Hence $k\left(x_{0}\right)=0$ for all $x_{0} \in X_{0}$, so that $k \equiv 0$, since $X_{0}$ is dense in $M$. This contradicts $k \neq 0$ in $K$, and completes the proof.

Lemmas 2 and 3 and the characterization of [5] give us:

THEOREM $4 . K$ is equivalent to $B(X)$ if and only if

(1) The structure space $M$ contains a dense set $X_{0}$ of isolated points.

(2) Every regular open set in $M$ is closed.

3. In [3] it was shown that $K=C(M)$ is equivalent to $B(X)$ if and only if either of the following sets of conditions is satisfied:

A. (1) $M$ contains a dense subset $X_{0}$ of isolated points.

(2) If $Q \leqq X_{0}$, then there exists an open and closed subset $S$ of $M$ such that $Q \leqq S$ and $S \cap X_{0}=Q$.

B. (1) For each $k \neq 0$ in $K$ there exists a minimal projection $e$ with $k e \neq 0$.

(2) For each subcollection $A$ of the collection $P$ of minimal projections, there exists a projection $e_{A}$ such that $e_{A} e_{p}=e_{p}$ for $p \in A$ and $e_{A} e_{p}=0$ for $p \notin A$.

The conditions of $\mathrm{A}$ are equivalent to those of $\mathrm{B}$ directly. This is essentially proved in Theorem 2 of [3]. That the (1)'s are equivalent is pointed out in our Lemma 3. The (2)'s, for example, are each obviously equivalent to the statement: If $A$ is a subset of $X_{0}$, there exists a function $e(x)$ in $C(M)$ such that $e(x)=1$ if $x \in A$, and $e(x)=0$ if $x \in X_{0}-A$.

Now assume that the conditions of Theorem 4 hold and let $Q \leqq X_{0}$. Then $Q$ is open and $Q \leqq$ int $\bar{Q} \leqq \bar{Q}$. But since each subset of $X_{0}$ is open (as a union of points which are open) it is also closed in the relative topology of $X_{0}$. Hence $Q=X_{0} \cap \bar{Q}$ and this implies $Q=X_{0} \cap$ int $\bar{Q}$. Then $S=$ int $\bar{Q}$ is open and closed (being a regular open set) and the conditions of $A$ are satisfied.

Now assume the conditions of $A$, and let $S$ be a regular open set in $M$. Let $Q$ be the complement of $X_{0} \cap S$ in $X_{0}$. Then $\left(\overline{X_{0} \cap S}\right) \cup \bar{Q}$ $=\bar{X}_{0}=M$. Now by (2) of $A$, there exists an open and closed set $T$ such that $X_{0} \cap S \leqq T$, but $Q \leqq T^{\prime}$. Thus $\overline{X_{0} \cap S} \leqq T, \bar{Q} \leqq T^{\prime}$, and $\left(\overline{X_{0} \cap S}\right) \cap \bar{Q}=0$, and $\overline{X_{0} \cap S}$ and $\bar{Q}$ are open, as well as closed. Now since $S \cap Q=0$ and $S$ is open, $S \cap \bar{Q}=0$. Since $\bar{Q}$ is open $\bar{S} \cap \bar{Q}=0$. Hence $\bar{S} \leqq \bar{Q}^{\prime}=\overline{X_{0} \cap S}$. Thus $\bar{S}=\overline{X_{0} \cap S}$ and $\bar{S}$ is open. Then $S=\operatorname{int}(\bar{S})$ $=\bar{S}$, and $S$ is closed. Hence the conditions of Theorem 4 are equivalent to $A$ and $B$. 


\title{
REFERENCES
}

1. I. Gelfand, Normierte Ringe, Rec. Math. (Mat. Sbornik) N.S. vol. 9 (1941) pp. 3-24.

2. I. Gelfand and M. Neumark, On the embedding of normed rings into the ring of operators in Hilbert Space, Rec. Math. (Mat. Sbornik) N.S. vol. 12 (1943) pp. 197213.

3. L. J. Heider, A note on a theorem of K. G. Wolfson, Proc. Amer. Math. Soc. vol. 6 (1955) pp. 305-308. 1943.

4. N. Jacobson, The theory of rings, Mathematical Surveys, vol. 2, New York,

5. K. G. Wolfson, The algebra of bounded functions, Proc. Amer. Math. Soc. vol. 5 (1954) pp. 10-14.

\section{RUTGERS UNIVERSITY}

\section{LIE SIMPLICITY OF A SPECIAL CLASS OF ASSOCIATIVE RINGS ${ }^{1}$}

\author{
WILLARD E. BAXTER
}

Given an associative ring $A$, by introducing a new multiplication we can form from it a new ring called the Lie ring of $A$. This multiplication is defined by $[a, b]=a b-b a$ for all $a, b \in A$. If $U$ is an additive subgroup of $A$ and if for arbitrary $u \in U, a \in A, u a-a u \in U$, then $U$ is said to be a Lie ideal of $A$. If $X, Y$ are additive subgroups of $A$ then by $[X, Y]$ we mean the additive subgroup generated by all the elements $x y-y x$, where $x \in X, y \in Y$. An additive subgroup $U$ of $[A, A]$ is said to be a proper Lie ideal of $[A, A]$ if $U \neq[A, A]$ and if $[U$, $[A, A]] \subset U$.

In [4], Herstein proved that if $A$ is a simple ring of characteristic not 2 or 3 , and if $U$ is a proper Lie ideal of $[A, A]$, then $U$ is contained in $Z$, the center of $A$. In this paper we settle the question in the open case where $A$ is a simple ring of characteristic 2 or 3 . The above theorem becomes sharpened to:

THEOREM 1. If $A$ is a simple ring and if $U$ is a proper Lie ideal of $[A, A]$, then $U$ is contained in the center of $A$, except for the case where $A$ is of characteristic 2 and 4 dimensional over its center, a field of characteristic 2.

Presented to the Society, October 22, 1955; received by the editors September 26, 1955.

${ }_{1}^{1}$ The results of this paper will comprise the beginning portion of a thesis, which will be presented to the Faculty of the Graduate School of the University of Pennsylvania in partial fulfillment of the requirements for the degree of Doctor of Philosophy. 